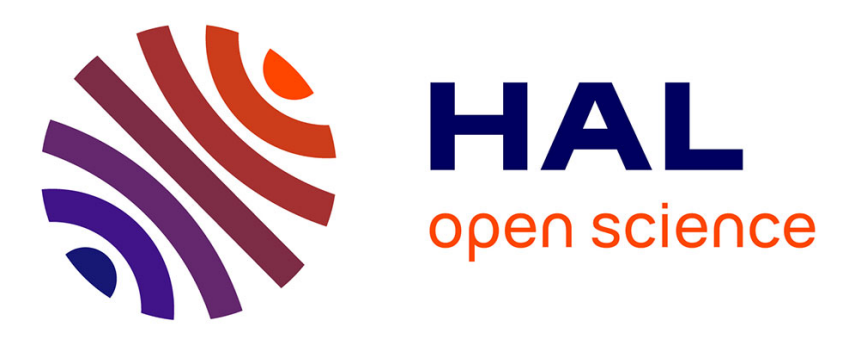

\title{
Nonlinear 2-DOFs Vibration Energy Harvester Based on Magnetic Levitation
}

\author{
Issam Abed, Najib Kacem, Mohamed Bouazizi, Noureddine Bouhaddi
}

\section{To cite this version:}

Issam Abed, Najib Kacem, Mohamed Bouazizi, Noureddine Bouhaddi. Nonlinear 2-DOFs Vibration Energy Harvester Based on Magnetic Levitation. International Modal Analysis Conference, Feb 2015, Orlando, FL, United States. hal-02963584

\section{HAL Id: hal-02963584 https://hal.science/hal-02963584}

Submitted on 10 Oct 2020

HAL is a multi-disciplinary open access archive for the deposit and dissemination of scientific research documents, whether they are published or not. The documents may come from teaching and research institutions in France or abroad, or from public or private research centers.
L'archive ouverte pluridisciplinaire HAL, est destinée au dépôt et à la diffusion de documents scientifiques de niveau recherche, publiés ou non, émanant des établissements d'enseignement et de recherche français ou étrangers, des laboratoires publics ou privés. 


\title{
Chapter 5 \\ Nonlinear 2-DOFs Vibration Energy Harvester Based on Magnetic Levitation
}

\begin{abstract}
The nonlinear dynamics of a two-degree-of-freedom (2-DOFs) vibrating energy harvester (VEH) based on 5 magnetic levitation is modeled and investigated. The equations of motion have been derived while taking into account 6 the magnetic nonlinearity and the electro-magnetic damping. The associated linear eigenvalue problem has been analyzed 7 and optimality conditions have been expressed in term of distance minimization between the two eigenfrequencies of the 8 considered system. The resulting optimal design parameters have been substituted into the coupled nonlinear equations of 9 motion which have been numerically solved. It is shown that the performances of a classical single degree of freedom VEH 10 can be significantly enhanced up to $270 \%$ in term of power density, up to $34 \%$ in term of frequency bandwidth and up to 11 $10 \%$ in term of resonance frequency attenuation.

Keywords Energy harvesting - Nonlinear dynamics $•$ Magnetic levitation $\bullet$ Electro-magnetic damping $\bullet$ Frequency 13 bandwidth

\subsection{Introduction}

Over recent years, many research activities are oriented to the development of some harvester devices to produce 16 inexhaustible electric energy in their local environment. The purpose in using Vibration energy harvesters (VEHs) is related 17 to the reduction of power requirement as well as the communication in an autonomous way and to the disposition of the 18 monitor, the measure, and the process data in a hostile environment. On this concept, VEHs can be used in many fields such 19 as environmental monitors, wireless sensors and medical implants [1-3]. In order to make VEHs usable, there are several 20 types of transduction, where the most common transduction modes are piezoelectric [4], electrostatic, magnetostrictive and 21 electromagnetic [5] and each of them presents advantages and disadvantages.

The linear VEHs has a low bandwidth and designed to collect power in the ambient dominant frequency. When the 23 excitation and resonance frequency of the system do not coincide, linear VEHs are known to under perform. So, to increase 24 the collected power and the bandwidth frequency, researches are oriented towards the study of nonlinear systems. For 25 instance, Daqaq et al. [6] reported softening frequency response characteristics in a parametrically forced piezoelectric device 26 with structural nonlinearities. Mann et al. [7] showed analytically and experimentally how magnetic levitation could be used 27 to extend device bandwidth through a hardening response. Nevertheless, this extension is limited by dry friction dissipation 28 phenomenon. Mahmoudi et al. [8] proposed an alternative to overcome this issue by guiding the moving magnet vertically 29

I. Abed

Applied Mechanics Department, FEMTO-ST Institute, UMR 6174, University of Franche-Comté, 24 chemin de 1'Epitaphe,

25000 Besançon, France

Preparatory Engineering Institute of Nabeul (IPEIN), 8000 M'Rezgua, Nabeul, Tunisia

Tunis El Manar University (UTM), El Manar, Tunisia

N. Kacem $(\bowtie) \cdot$ N. Bouhaddi

Applied Mechanics Department, FEMTO-ST Institute, UMR 6174, University of Franche-Comté, 24 chemin de 1'Epitaphe, 25000 Besançon, France

e-mail: najib.kacem@femto-st.fr

M.L. Bouazizi

Preparatory Engineering Institute of Nabeul (IPEIN), 8000 M'Rezgua, Nabeul, Tunisia

Tunis El Manar University (UTM), El Manar, Tunisia

Mechanical Department, College of Engineering Salman Bin Abdulaziz University, Al Kharj, Saudi Arabia

(C) Springer International Publishing Switzerland 2015

A. Wicks (ed.), Shock \& Vibration, Aircraft/Aerospace, and Energy Harvesting, Volume 9 ,

Conference Proceedings of the Society for Experimental Mechanics Series, DOI 10.1007/978-3-319-15233-2_5 


\section{Author's Proof}

in an elastic way by means of sandwich beams, combined with an hybrid piezoelectric and electromagnetic transductions. 30 Although their numerical results demonstrated high performances, the electronic circuit may become very complex. 31

In this paper, we propose a design of a VEH based on the magnetic levitation of two coupled magnets. The equations 32 of motion have been derived and include the magnetic nonlinearity and the electro-magnetic damping. While respecting 33 the optimality conditions expressed in term of distance minimization between the two eigenfrequencies of the associated 34 linear problem, the coupled nonlinear equations of motion have been solved numerically using the harmonic balance method 35 (HBM) [9] coupled with the asymptotic numerical continuation technique (ANM) [10]. We prove numerically that the 36 nonlinear coupling between the magnets permits the performance enhancement of a classical single degree of freedom VEH 37 in terms of frequency attenuation, power density and frequency bandwidth.

\subsection{Design and System Modeling}

\subsubsection{Proposed Device}

Inspired by [5, 7], an extension of magnetic levitation for a multi-degree of freedom vibration energy harvester is proposed. 41 As a proof of concept, the considered device shown in Fig. 5.1 is composed of four magnets $M_{i}$ where $i \in[1,4]$. $M_{1}$ and 42 $M_{4}$ are fixed with respect to a Teflon tube inside which $M_{2}$ and $M_{3}$ are subjected to magnetic levitation forces. All magnets 43 are placed vertically in such a way that all opposed surfaces have the same pole and wire-wound copper coils are wrapped 44 horizontally around the separation distance between each two adjacent magnets.

Three reference frames have been applied in order to describe the electromagnetic induction model represented in Fig. 5.1. 46 The first reference frame is fixed in space and is used to describe the motion amplitude $Y_{0}$ and excitation frequency $\Omega$ of the 47 outer housing. The second and third reference frames (designed as $x_{2}$ and $x_{3}$ ) describe the motion of the moving magnets 48 $M_{2}$ and $M_{3}$ which are subjected to restoring forces expressed as follows:

$$
\begin{aligned}
& \mathbf{F}_{(2)}^{m}=\left(-\frac{M_{2} g d_{0}^{2}}{\left(M_{1} Q_{1}-M_{3} Q_{3}\right)}\left(\frac{M_{1} Q_{1}}{\left(d_{0}-v_{2}\right)^{2}}-\frac{M_{3} Q_{3}}{\left(d_{0}+v_{2}-v_{3}\right)^{2}}\right)+M_{2} g\right) \overrightarrow{\mathbf{x}_{2}} \\
& \mathbf{F}_{(3)}^{m}=\left(-\frac{M_{3} g d_{0}^{2}}{\left(M_{2} Q_{2}-M_{4} Q_{4}\right)}\left(\frac{M_{3} Q_{3}}{\left(d_{0}+v_{2}-v_{3}\right)^{2}}-\frac{M_{4} Q_{4}}{\left(d_{0}+v_{3}\right)^{2}}\right)+M_{3} g\right) \overrightarrow{\mathbf{x}}_{3}
\end{aligned}
$$

When the device is subjected to an external mechanical vibration, the moving magnets oscillate around their equilibrium 50 positions and a current is induced in each coil as shown in Fig. 5.2, resulting in the following electrical damping forces:

$$
\begin{aligned}
& F_{(2)}^{e}=c e_{1} \dot{v}_{2}-c e_{2}\left(\dot{v}_{3}-\dot{v}_{2}\right) \\
& F_{(3)}^{e}=c e_{3} \dot{v}_{3}-c e_{2}\left(\dot{v}_{2}-\dot{v}_{3}\right)
\end{aligned}
$$

Fig. 5.1 A schematic diagram of the two degree of freedom vibration energy harvester based on magnetic levitation

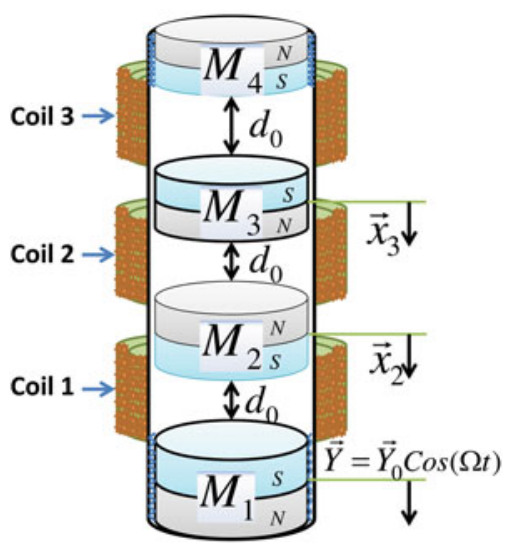




\section{Author's Proof}

5 Nonlinear 2-DOFs Vibration Energy Harvester Based on Magnetic Levitation

Fig. 5.2 Equivalent electro-mechanical system of the two degree of freedom vibration energy harvester based on magnetic levitation

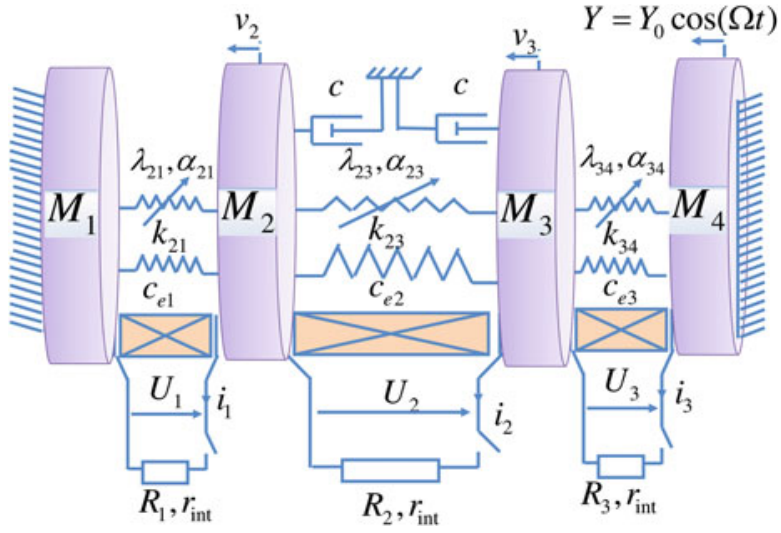

The electrical damping can be expressed as a function of the internal resistance of the coil, the resistance of external load 53 and the electromechanical coupling coefficient.

$$
c e_{j}=\frac{\alpha_{j}^{2}}{R_{j}+r_{i n t}}
$$

where $\alpha_{j}=N_{j} B l$, with $B$ the average magnetic field strength, $N_{j}$ is the number of coil turns and $l$ is the coil length.

Expanding the non-linear magnetic forces written in Eq. (5.2) in Taylor series up to the third order and summing all 56 forces applied to each moving magnet in the vertical direction, we obtain the following governing equations of motion for 57 the mechanical system:

$$
\left\{\begin{array}{l}
\left(\begin{array}{l}
M_{2} \ddot{v}_{2}+\left(c+c e_{1}+c e_{2}\right) \dot{v}_{2}-c e_{2} \dot{v}_{3}+k_{23}\left(v_{2}-v_{3}\right)+k_{21}\left(v_{2}\right) \\
+\alpha_{23}\left(v_{2}-v_{3}\right)^{2}-\alpha_{21}\left(v_{2}\right)^{2}+\lambda_{23}\left(v_{2}-v_{3}\right)^{3}+\lambda_{21}\left(v_{2}\right)^{3}
\end{array}\right)=-M_{2} \ddot{Y} \\
\left(\begin{array}{l}
M_{3} \ddot{v}_{3}+\left(c+c e_{2}+c e_{3}\right) \dot{v}_{3}-c e_{2} \dot{v}_{2} \\
+k_{34}\left(v_{3}\right)+k_{32}\left(v_{3}-v_{2}\right)+\alpha_{34}\left(v_{3}\right)^{2} \\
-\alpha_{32}\left(v_{3}-v_{2}\right)^{2}+\lambda_{34}\left(v_{3}\right)^{3}+\lambda_{32}\left(v_{3}-v_{2}\right)^{3}
\end{array}\right)=-M_{3} \ddot{Y}
\end{array}\right.
$$

If the magnetic intensities (Q) are equal, $\forall j \in[2,3]$ the linear and nonlinear stiffnesses can be written as:

$$
\begin{gathered}
k_{j(j+1)}=\frac{2 g M_{j} M_{j+1}}{\left(M_{j-1}-M_{j+1}\right) d_{0}} ; k_{j(j-1)}=\frac{2 g M_{j} M_{j-1}}{\left(M_{j-1}-M_{j+1}\right) d_{0}} \\
\alpha_{j(j+1)}=\frac{3}{2 d_{0}} k_{j(j+1)} ; \alpha_{j(j-1)}=\frac{3}{2 d_{0}} k_{j(j-1)} \\
\lambda_{j(j+1)}=\frac{2}{d_{0}^{2}} k_{j(j+1)} ; \lambda_{j(j-1)}=\frac{2}{d_{0}^{2}} k_{j(j-1)}
\end{gathered}
$$

\subsection{Optimality Conditions and Average Power}

Firstly, the associated linear problem is written in its matrix form. Then, in order to guaranty the symmetry of the rigidity 61 matrix, we assume that $k_{23}=k_{32}$ which results in the following relation between the magnets in term of mass:

$$
M_{4}+M_{1}=M_{2}+M_{3}
$$

While respecting Eq. (5.8), the eigen frequencies can be written as follows:

$$
\Omega_{1}{ }^{2}, \Omega_{2}{ }^{2}=\left(\frac{1}{2}\left\{\frac{\left(k_{23}+k_{21}\right) M_{3}+\left(k_{34}+k_{32}\right) M_{2}}{M_{2} M_{3}}\right\} \pm \frac{1}{2} \sqrt{\left[\begin{array}{l}
\left\{\frac{\left(k_{23}+k_{21}\right) M_{3}+\left(k_{34}+k_{32}\right) M_{2}}{M_{2} M_{3}}\right\}^{2} \\
-4\left\{\frac{\left(k_{23}+k_{21}\right)\left(k_{34}+k_{32}\right)-k_{23} k_{32}}{M_{2} M_{3}}\right\}
\end{array}\right]}\right)
$$




\section{Author's Proof}

In order to enhance the performances of the considered VEH in term of bandwidth, we minimize the distance separating 64 the naturel frequencies expressed in Eq. (5.9). Doing so, we obtain the following optimality conditions:

$$
\left\{\begin{array}{l}
M_{2}=M_{1}-M_{4} \\
M_{3}=2 M_{4} \\
M_{1}>2 M_{4}
\end{array}\right.
$$

The optimality conditions (5.10) have been substituted in the system of nonlinear equations (5.6) which have been solved 66 numerically using the harmonic balance method coupled with the asymptotic numerical continuation technique. Thus, the 67 nonlinear frequency responses can be plotted in term of velocity that will be used to determine the average power delivered 68 to the electrical load.

Indeed, the magnetic transduction is ensured by three coils. The oscillations of the movable magnets cause magnetic field 70 variations in the separation zones, which provides an induced current (Lenz's Law). The induced current can be expressed 71 as a vibration velocity function $\dot{v}_{j}(t)=V_{j} \Omega \sin (\Omega t) ; \forall j \in[2,3]$. Then, the average power delivered to the electrical load 72 can be written as follows:

$$
P_{m}=\frac{1}{T} \int_{0}^{T} P(t) d t=\frac{\Omega^{2}}{2}\left(\sum_{j=1}^{3}\left(c e_{j}\left(V_{j+1}-V_{j}\right)^{2}\right)\right)
$$

\subsection{Results and Discussion}

\subsubsection{Optimal Design Parameters}

In order to enhance the bandwidth of the proposed device, the distance between the eigenfrequencies written in Eq. (5.9) is 76 plotted with respect to $M_{1}$ and $M_{4}$ as shown in Fig. 5.3. Remarkably, Eq. (5.10) are not verified for regions 1 and 2 which are 77 separated by an optimal zone for which $f_{2}-f_{1}<4.3 \mathrm{~Hz}$. Inside this region, a set of design parameters, listed in Table 5.1, 78 is chosen.

Fig. 5.3 Variation of the distance separating the eigenfrequencies of the proposed VEH with respect to $M_{1}$ and $M_{4}$. Inside the optimal zone, $f_{2}-f_{1}<4.3 \mathrm{~Hz}$

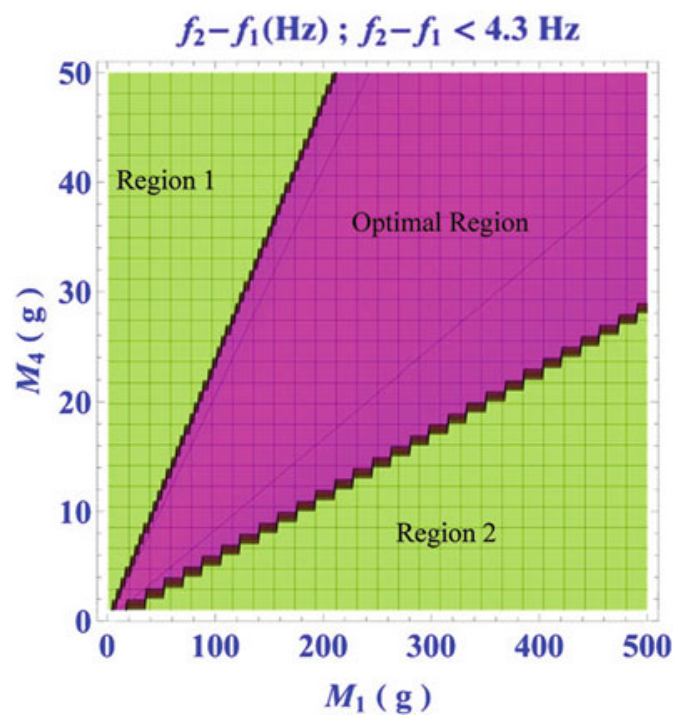


Author's Proof

5 Nonlinear 2-DOFs Vibration Energy Harvester Based on Magnetic Levitation

Table 5.1 Physical and geometric properties of the proposed 2-DOFs vibration energy harvester

\begin{tabular}{|c|c|c|}
\hline$r_{\text {int }}$ & Internal resistance $(\Omega)$ & 188 \\
\hline$l$ & Total coil length (m) & 0.015 \\
\hline$d_{0}$ & Separation distance $(\mathrm{m})$ & 0.015 \\
\hline$B$ & Residual magnetic flux density (T) & 1.18 \\
\hline$c$ & Mechanical damping [7] $(\mathrm{Ns} / \mathrm{m})$ & 0.116 \\
\hline$\rho_{m}$ & Magnet density $\left(\mathrm{kg} / \mathrm{m}^{2}\right)$ & 7800 \\
\hline$R_{j}, j \in[1,3]$ & External load resistance $(\Omega)$ & $10^{4}$ \\
\hline$N_{j}, j \in[1,3]$ & The coil number (turns) & 2000 \\
\hline$M_{1}$ & Mass $(k g)$ & 0.125 \\
\hline$M_{2}$ & Mass $(k g)$ & 0.0195 \\
\hline$M_{3}$ & Mass $(k g)$ & 0.02 \\
\hline$M_{4}$ & Mass $(k g)$ & 0.01 \\
\hline
\end{tabular}

Fig. 5.4 Variation of the frequency responses in term of harvested power with respect to the excitation amplitude for the proposed device. Black and gray lines denote respectively stable and unstable branches

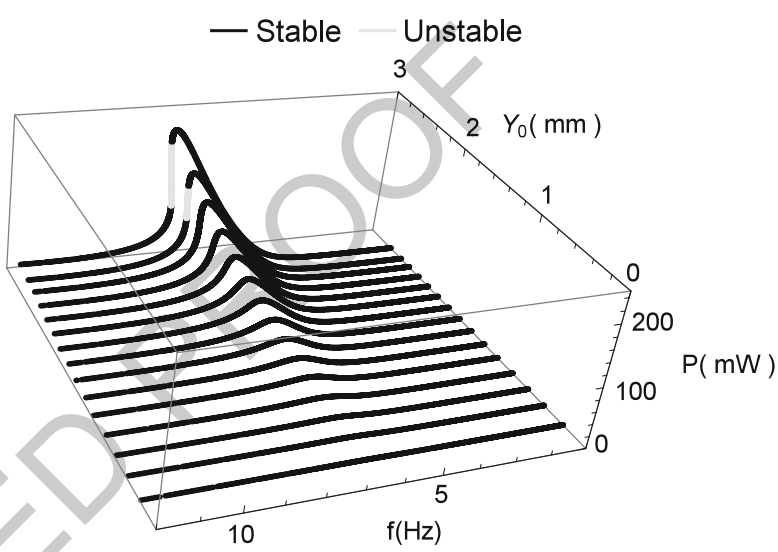

Fig. 5.5 A typical frequency response of the proposed device showing a hardening behavior for an excitation amplitude

$Y_{0}=2.7 \mathrm{~mm}$, the frequency bandwidth is $B W=14.4 \%$. Solid and dashed lines denote respectively stable and unstable branches

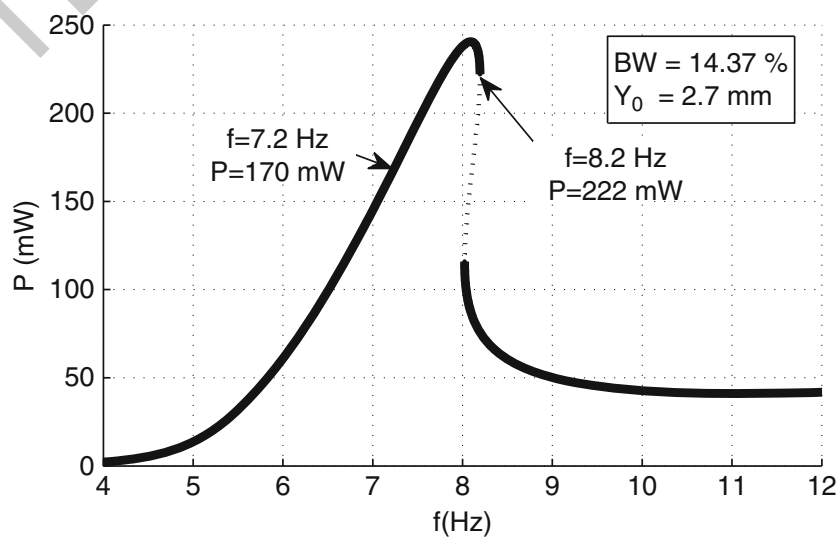

\subsubsection{Bandwidth Enhancement}

Several numerical simulations have been performed for the set of design parameters listed in Table 5.1. Figure 5.4 displays 81 the evolution of the frequency response in term of harvested power for different values of amplitude excitation $Y_{0}$ up to 82 $3 \mathrm{~mm}$. It is shown that bistability takes place for large excitation amplitudes $\left(Y_{0}>2 \mathrm{~mm}\right)$ for which the dynamic response 83 has two solutions for a given frequency inside a range separated by two bifurcation points.

Hence, one can take advantage of the nonlinear spring hardening effect in order to enlarge the frequency bandwidth of the 85 VEH. Interestingly, Fig. 5.5 shows that the frequency bandwidth of the proposed device can reach $14.4 \%$ for an excitation 86 amplitude $Y_{0}=2.7 \mathrm{~mm}$. 


\section{Author's Proof}

I. Abed et al.

Fig. 5.6 Variation of the frequency responses in term of normalized power density with respect to the excitation amplitude for the proposed device. Black and gray lines denote respectively stable and unstable branches

Fig. 5.7 Comparison of the frequency responses in term of normalized power density between the proposed device (2-DOFs) and the single degree of freedom VEH presented in [7]

Table 5.2 Performances of 1-DOF and 2-DOFs vibration energy harvesters based on magnetic levitation
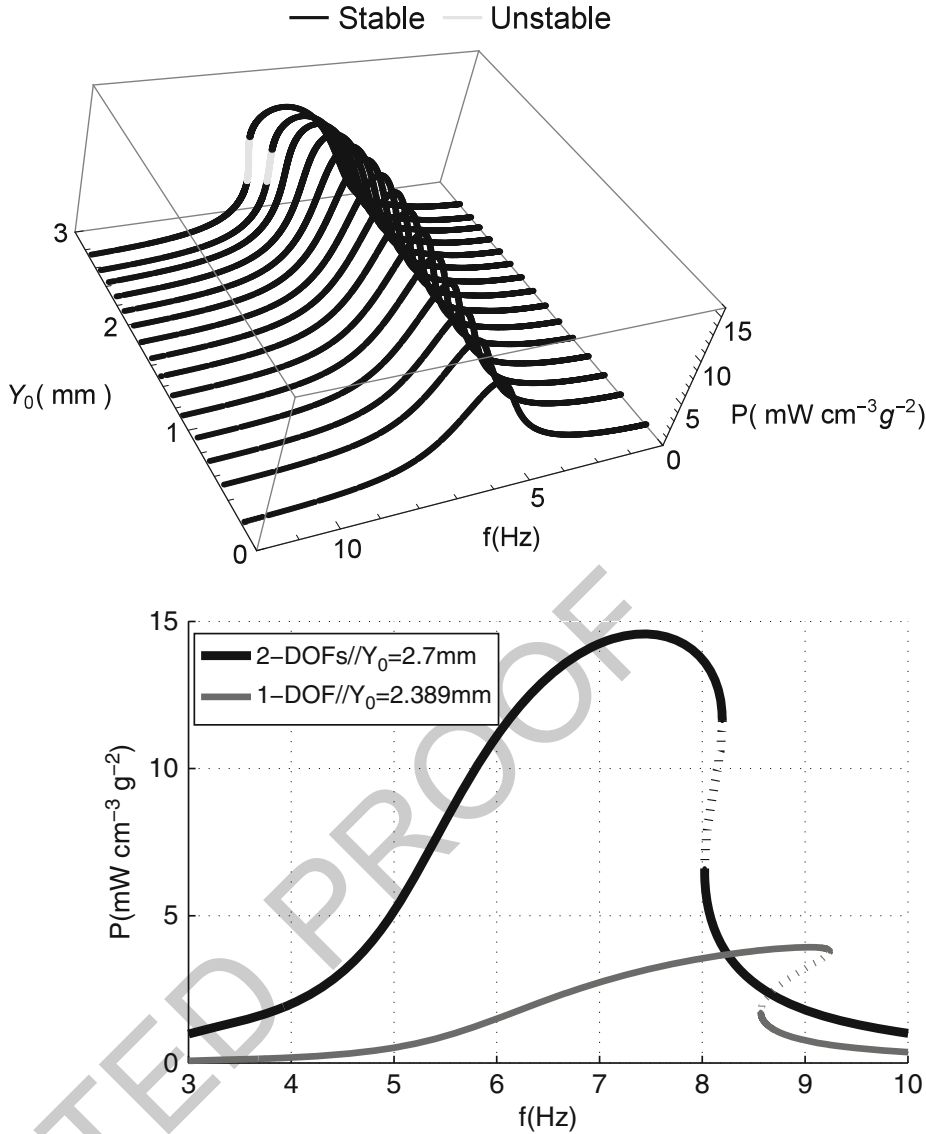

\begin{tabular}{|c|c|c|c|c|}
\hline Model & $\begin{array}{l}\text { Resonance frequency } \\
(\mathrm{Hz})\end{array}$ & $\begin{array}{l}\text { Frequency bandwidth } \\
(\%)\end{array}$ & $\begin{array}{l}\text { Normalized power } \\
\left(\mathrm{mWcm}^{-3} \mathrm{~g}^{-2}\right)\end{array}$ & $\begin{array}{l}\text { Power } \\
(\mathrm{mW})\end{array}$ \\
\hline 1-DOF & 6.96 & 10.5 & 3.93 & 35.38 \\
\hline 2-DOFs & 6.60 & 14.4 & 14.57 & 240.50 \\
\hline
\end{tabular}

\subsubsection{Normalized Power Density Enhancement}

Since the harvested power varies with respect to the excitation amplitude and in order to compare the proposed device with 89 the one investigated in [7], in terms of performances, we propose the use of the normalized power density as a kind of 90 energetic efficiency. The latter varies slightly with respect to $Y_{0}$ which is proved in Fig. 5.6 showing that the maximum of 91 frequency curves is almost constant independently of the excitation amplitude.

Figure 5.7 shows that the energetic efficiency of a single degree of freedom VEH based on magnetic levitation can be 93 enhanced up to $270 \%$ by coupling two moving magnets. Moreover, the bandwidth can be boosted up to $34 \%$ and the 94 frequency attenuation is about $10 \%$ as listed in Table 5.2.

\subsection{Conclusion}

The non-linear dynamics of a two-degree-of-freedom vibrating energy harvester (VEH) was modeled including the main 97 sources of non-linearities. The associated linear eigen problem was analytically solved and optimality conditions were 98 derived in term of distance minimization between the two eigenfrequencies. The resulting coupled nonlinear equations of 99 motion were solved numerically for the optimal design, using the harmonic balance method coupled with the asymptotic 100 numerical continuation technique. 
Author's Proof

5 Nonlinear 2-DOFs Vibration Energy Harvester Based on Magnetic Levitation

Several numerical simulations have been performed to highlight the performance of the proposed device. Particularly, 102 the power density, the bandwidth and the frequency attenuation can be boosted up to $270 \%$, 34\% and $10 \%$ respectively 103 compared to the case of a single degree of freedom magnetic levitation based VEH, thanks to the nonlinear coupling between 104 the magnets. Future work will include the extension of the proposed concept to large arrays of coupled levitated magnets.

\section{References}

1. Ding Z, Perlaza SM, Esnaola I, Poor HV (2013) Power allocation strategies in energy harvesting wireless cooperative networks. IEEE Trans 107 Wireless Commun 13:846-860

2. Donelan JM, Li Q, Naing V, Hoffer JA, Weber DJ, Kuo AD (2008) Biomechanical energy harvesting: generating electricity during walking 109 with minimal user effort. Science 319:807-810

3. Wang W, Wang N, Jafer E, Hayes M, O’Flynn B, O’Mathuna C (2010) Autonomous wireless sensor network based building energy 111 and environment monitoring system design. In: Environmental Science and Information Application Technology (ESIAT), International 112 Conference

4. Zhou L, Sun J, Zheng XJ, Deng SF, Zhao JH, Peng ST, Zhang Y, Wang XY, Cheng HB (2012) A model for the energy harvesting performance 114 of shear mode piezoelectric cantilever. Sens Actuators A Phys 179:185-192

5. Foisal ARM, Hong C, Chung GS (2012) Multi-frequency electromagnetic energy harvester using a magnetic spring cantilever. Sens Actuators 116 A Phys 182:106-113

6. Daqaq MF, Stabler C, Qaroush Y, Seuaciuc-Osório T (2009) Investigation of power harvesting via parametric excitations. J Intel Mater Syst 118 Struct 20(5):545-557

7. Mann BP, Sims ND (2009) Energy harvesting form the nonlinear oscillations of magnetic levitation. J Sound Vib 15(319):515-530

8. Mahmoudi S, Kacem N, Bouhaddi N (2014) Enhancement of the performance of a hybrid nonlinear vibration energy harvester based on 121 piezoelectric and electromagnetic transductions. Smart Mater Struct 23(7):075024

9. Cochelin B, Vergez C (2009) A high order purely frequency-based harmonic bal-ance formulation for continuation of periodic solutions. 123 J Sound Vib 324:243-262

10. Arquier R, Karkar S, Lazarus A, Thomas O, Vergez C, Cochelin B, Manlab:an interactive path-following and bifurcation analysis software. 125 /http://manlab.lma.cnrs-mrs.fr/s 